\title{
ALDH1 and podoplanin expression patterns predict the risk of malignant transformation in oral leukoplakia
}

\author{
UMMA HABIBA $^{1}$, KYOKO HIDA ${ }^{2}$, TETSUYA KITAMURA ${ }^{1}$, AYA YANAGAWA MATSUDA ${ }^{1}$, \\ FUMIHIRO HIGASHINO ${ }^{1}$, YOICHI M. ITO ${ }^{3}$, YOICHI OHIRO ${ }^{4}$, \\ YASUNORI TOTSUKA ${ }^{4}$ and MASANOBU SHINDOH ${ }^{1}$
}

\author{
${ }^{1}$ Department of Oral Pathology and Biology, Hokkaido University Graduate School of Dental Medicine; \\ ${ }^{2}$ Department of Vascular Biology, Frontier Research Unit, Institute for Genetic Medicine, Hokkaido University; \\ ${ }^{3}$ Department of Biostatistics, Hokkaido University Graduate School of Medicine; ${ }^{4}$ Department of Oral and \\ Maxillofacial Surgery, Hokkaido University Graduate School of Dental Medicine, Sapporo 060-0815, Japan
}

Received June 22, 2015; Accepted October 24, 2016

DOI: $10.3892 / \mathrm{ol} .2016 .5379$

\begin{abstract}
Oral leukoplakia (OL) is a clinically diagnosed preneoplastic lesion of the oral cavity with an increased oral cancer risk. However, the risk of malignant transformation is still difficult to assess. The objective of the present study was to examine the expression patterns of aldehyde dehydrogenase 1 (ALDH1) and podoplanin in OL, and to determine their roles in predicting oral cancer development. In the present study, the expression patterns of ALDH1 and podoplanin were determined in samples from 79 patients with OL. The association between protein expression and clinicopathological parameters, including oral cancer-free survival, was analyzed during a mean follow-up period of 3.4 years. Expression of ALDH1 and podoplanin was observed in 61 and $67 \%$ patients, respectively. Kaplan-Meier analysis demonstrated that the expression of the proteins was correlated with the risk of progression to oral cancer. Multivariate analysis revealed that expression of ALDH1 and podoplanin was associated with 3.02- and 2.62-fold increased risk of malignant transformation, respectively. The malignant transformation risk of OL was considerably higher in cases with expression of both proteins. Point-prevalence analysis revealed that $66 \%$ of patients with co-expression of ALDH1 and podoplanin developed oral cancer. Taken together, our data indicate that ALDH1 and podoplanin expression patterns in OL are associated with oral cancer development, suggesting that ALDH1 and podoplanin may be useful biomarkers to identify OL patients with a substantially high oral cancer risk.
\end{abstract}

Correspondence to: Dr Kyoko Hida, Department of Vascular Biology, Frontier Research Unit, Institute for Genetic Medicine, Hokkaido University, N15, W7, Kita-Ku, Sapporo 060-0815, Japan E-mail:khida@igm.hokudai.ac.jp

Key words: oral leukoplakia, malignant transformation, ALDH1, podoplanin, cancer stem cell

\section{Introduction}

Oral cancer is one the leading causes of cancer mortality worldwide, and early diagnosis of high-risk, potentially malignant lesions is a high priority for reducing both morbidity and mortality (1-3). Oral leukoplakia (OL) is a clinically diagnosed preneoplastic lesion of the oral cavity with a frequency of malignant transformation of $17-24 \%$ (4-6). However, the risk of malignant transformation of OL is still difficult to assess. Although lesions with dysplastic features are considered to be at a higher risk for malignant transformation, the majority of oral cancers develop from lesions that lack dysplastic changes $(4,5)$. Therefore, objective biomarkers are required to evaluate the risk of malignant transformation in OL as well as for prophylactic intervention and proper management of the high-risk patient group. The cancer stem cell theory indicates that the initiation, progression, recurrence and metastasis of head and neck squamous cell carcinoma (HNSCC) are related to the behavior of a small subpopulation of cancer stem cells (7-9). The identification of these cells is important, not only for a better understanding of early detection and progression of cancer, but also for the development of more effective therapies. Diverse cell surface markers have been used for the identification of cancer stem cells in human tumors (10). ALDH1 and podoplanin are two well-studied cancer stem cell markers that have been implicated in several solid tumor types, including oral cancer $(11,12)$.

ALDH1 is an isoform of aldehyde dehydrogenase, which is expressed in humans as a cytosolic detoxifying isoenzyme that oxidizes intracellular aldehydes and contributes to the oxidation of retinol to retinoic acid in early stem cell differentiation (13). Previous studies have shown that ALDH1 is a specific marker for the identification of head and neck cancer stem cells, and serves a crucial role in maintaining the self-renewal properties and tumorigenicity of HNSCC-derived cancer stem cells $(11,14-16)$. Furthermore, ALDH1 overexpression was observed in oral dysplasia (17), and it was suggested to be a promising biomarker for distinguishing malignant from premalignant OL (17) and other oral dysplasia (18). In addition, ALDH1 immunoexpression 
is present in tissue samples of human epithelial cancer, including HNSCC, and is a prognostic marker for HNSCC survival (19-21).

Podoplanin is a mucin-type transmembrane glycoprotein that is specifically expressed in lymphatic endothelial cells but not in blood endothelial cells (22). High podoplanin expression was reported in HNSCC with lymph node metastasis and poor survival (23). Wicki and Christofori (12) suggested that podoplanin may act as a mediator of tumor cell invasion and metastasis. In addition, overexpression of podoplanin in $\mathrm{OL}$ and lichen planus suggested the use of podoplanin as a biomarker for oral cancer risk in patients with oral premalignancy $(24,25)$. Podoplanin is also expressed in a number of hyperplastic and dysplastic areas adjacent to the primary oral cancer, indicating that its abnormal expression occurs early in oral tumorigenesis, and may serve a role in malignant transformation (26). In the present study, it was hypothesized that the expression patterns of ALDH1 and podoplanin in OL would have predictive value for early detection of oral cancer. Therefore, the immunoexpression of ALDH1 and podoplanin was examined in samples from 79 patients with OL, and their usefulness as biomarkers for cancer risk assessment was evaluated.

\section{Materials and methods}

Patients and tissue specimens. All the medical records of 79 patients diagnosed with OL from Janauray 2002 to July 2012 at Hokkaido University Hospital (Sapporo, Japan) were retrieved and reviewed at the Department of Oral Pathology and Biology, Hokkaido University Graduate School of Dental Medicine (Sapporo, Japan). The clinical data were obtained from the medical records, while the biopsy specimens were obtained from formalin-fixed paraffin-embedded tissues. The expression pattern of ALDH1 and podoplanin was determined immunohistochemically in 79 OL patients during the follow-up period. The latency of the progressor was defined as the interval from the first biopsy of OL to subsequent OSCC development. A minimum of 6 months latency was maintained in all cases. Associations between the protein expression patterns and clinicopathological parameters, including oral cancer development during the follow-up period, were analyzed statistically. In the present retrospective follow-up study, malignant transformation vs. non-transformation was considered as a surrogate for clinical outcomes of patients with OL. The present study was approved by the institutional review board.

Histologic examination. Hematoxylin and eosin-stained slides were cut for routine diagnostics. The World Health Organization criteria (27) for OL and epithelial dysplasia were used when examining the histopathology of the sections. The presence of dysplasia was graded as mild, moderate or severe. Dysplasia was mild when dysplastic changes were present in less than a third of the epithelia, moderate when two thirds of the epithelia were affected and severe when the whole thickness of the epithelia was involved. During statistical analysis, mild dysplasia was considered as low-grade dysplasia (LGD), while moderate-to-severe dysplasia was considered as high-grade dysplasia (HGD).
Tissue processing and immunohistochemistry. Immunohistochemical staining was performed using the streptavidin-peroxidase methods described previously (28). In brief, immunostaining was conducted using $5-\mu \mathrm{m}$ paraffin-embedded tissue serial sections. The slides were deparaffinized in xylene, rehydrated in graded alcohol and subjected to antigen retrieval by heat treatment in Tris-EDTA buffer. To inhibit endogenous peroxidase activity, the slides were immersed in 3\% hydrogen peroxide for $5 \mathrm{~min}$, followed by blocking solution [1\% bovine serum albumin (A8327; Sigma-Aldrich; Merck Millipore, Darmstadt, Germany) in PBS) for $30 \mathrm{~min}$. The slides were then incubated with anti-ALDH1 (clone 44/ALDH1; 1:100 dilution; BD Transduction Laboratories ${ }^{\mathrm{TM}}$; BD Biosciences, Franklin Lakes, NJ, USA) and anti-podoplanin (clone D2-40; 1:100 dilution; Vector Laboratories, Inc., Burlingame, CA, USA) monoclonal antibodies at $4^{\circ} \mathrm{C}$ overnight. The sections were then treated with N-Histofine ${ }^{\circledR}$ Simple Stain Rat MAX PO (M) (Nichirei Bioscience, Inc., Tokyo, Japan) at $37^{\circ} \mathrm{C}$ for $30 \mathrm{~min}$. Visualization was conducted using ChemMate ${ }^{\mathrm{TM}}$ DAKO EnVision ${ }^{\mathrm{TM}} / \mathrm{HRP}$ (Dako North America, Inc., Carpinteria, CA, USA). Cytoplasmic and/or cell membrane immunoreactivity in the epithelium was considered to indicate positive ALDH1 and podoplanin expression, respectively. To analyze the prognostic value for cancer development, the ALDH1 and podoplanin expression levels were categorized as follows: Lesions with no expression or expression restricted to the basal layer of the oral epithelium were considered to be negative, while immunoexpression extending to the suprabasal layer of the oral epithelium was considered to be ALDH1 and podoplanin positive.

Statistical analysis. The associations between protein (ALDH1 and podoplanin) expression and clinicopathological variables were assessed using the Wilcoxon rank-sum test for continuously distributed variables and the $\chi^{2}$ test for categorical variables. Kaplan-Meier survival analysis was used to estimate the events of interest for oral cancer-free survival (OCFS) and the time interval from histopathological diagnosis to the development of oral squamous cell carcinoma (OSCC). Patients who did not develop invasive OSCC were censored at their last date of follow-up. The log-rank test was used to compare survival times among patients with different characteristics. The Cox regression model was applied to evaluate the hazard ratio (HR) for malignant transformation of OL. HRs with a 95\% confidence interval (CI) and P-values were reported. The likelihood ratio test was applied to evaluate the point-prevalence value (PPV). All tests were two sided, and $\mathrm{P}<0.05$ was considered to indicate a statistically significant difference. JMP ${ }^{\circledR}$ Pro version 10.0.2 (SAS Institute Inc., Cary, $\mathrm{NC}$, USA) was used for statistical analysis.

\section{Results}

Patient characteristics, and ALDHI and podoplanin expression. According to the follow-up record, patients primarily diagnosed as OL were grouped into untransformed (UT; n=42) and malignant transformed OL (MT; n=37) lesions. Patient with untransformed OL was defined as who did not develop into OSCC during the follow-up period, while patient with malignant transformed OL was defined as who 
Table I. Patients' baseline characteristics.

\begin{tabular}{|c|c|}
\hline Characteristics & $\mathrm{N}(\%)$ \\
\hline All patients & 79 \\
\hline \multicolumn{2}{|l|}{ Age, years } \\
\hline Mean \pm SD & $70 \pm 12$ \\
\hline Median & 72 \\
\hline \multicolumn{2}{|l|}{ Gender } \\
\hline Female & $54(68)$ \\
\hline Male & $25(32)$ \\
\hline \multicolumn{2}{|c|}{ Follow-up, months } \\
\hline Mean \pm SD & $42.1 \pm 34.1$ \\
\hline Median & 25 \\
\hline \multicolumn{2}{|l|}{ Site } \\
\hline Tongue & $28(35)$ \\
\hline Gingiva & $18(23)$ \\
\hline $\mathrm{BM}$ & $21(27)$ \\
\hline FOM & $5(6)$ \\
\hline Other & $7(9)$ \\
\hline \multicolumn{2}{|l|}{ Dysplasia } \\
\hline LGD & $27(34)$ \\
\hline HGD & $52(66)$ \\
\hline \multicolumn{2}{|c|}{ ALDH1 expression } \\
\hline Positive & $48(61)$ \\
\hline Negative & $31(39)$ \\
\hline \multicolumn{2}{|c|}{ Podoplanin expression } \\
\hline Positive & $53(67)$ \\
\hline Negative & $26(33)$ \\
\hline \multicolumn{2}{|c|}{ Co-expression of ALDH1 and podoplanin } \\
\hline Positive & $35(44)$ \\
\hline Negative & $44(56)$ \\
\hline \multicolumn{2}{|c|}{ Expression of either ALDH1 or podoplanin } \\
\hline Positive & $66(84)$ \\
\hline Negative & $13(16)$ \\
\hline \multicolumn{2}{|c|}{ Malignant transformation } \\
\hline Yes & $37(47)$ \\
\hline No & $42(53)$ \\
\hline
\end{tabular}

SD, standard deviation; ALDH1, aldehyde dehydrogenase 1; LGD, low-grade dysplasia; HGD, high-grade dysplasia; BM, buccal mucosa; FOM, floor of the mouth.

subsequently developed into OSCC during the follow-up period. Patients' baseline characteristics and associations between expression of the proteins (ALDH1 and podoplanin) and clinicopathological parameters are summarized in Tables I and II, respectively. A significant difference between protein expression patterns and degree of dysplasia was noted, whereas differences in age, gender or lesion site were not significant.

The expression patterns of ALDH1 and podoplanin in normal epithelium and serial tissue samples of OL are illustrated in Fig. 1. Among the 79 cases, 48 (61\%) and $53(67 \%)$

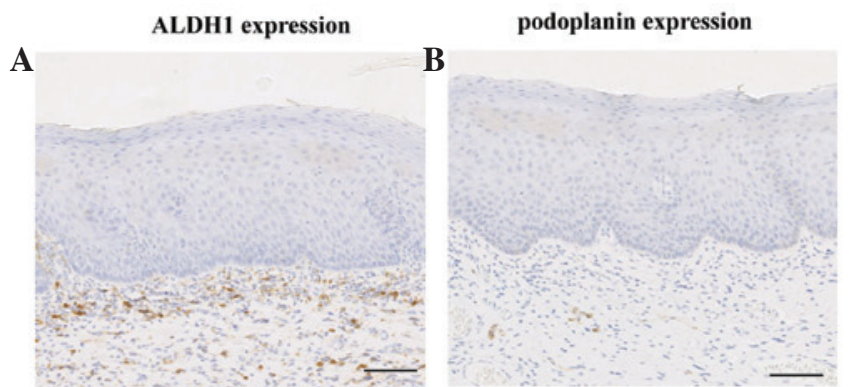

C
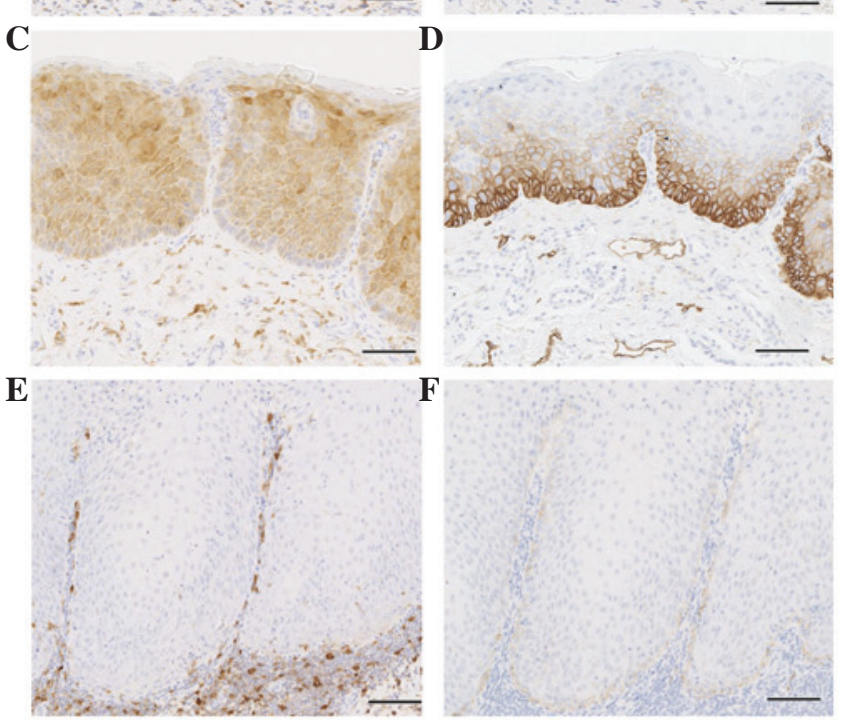

G
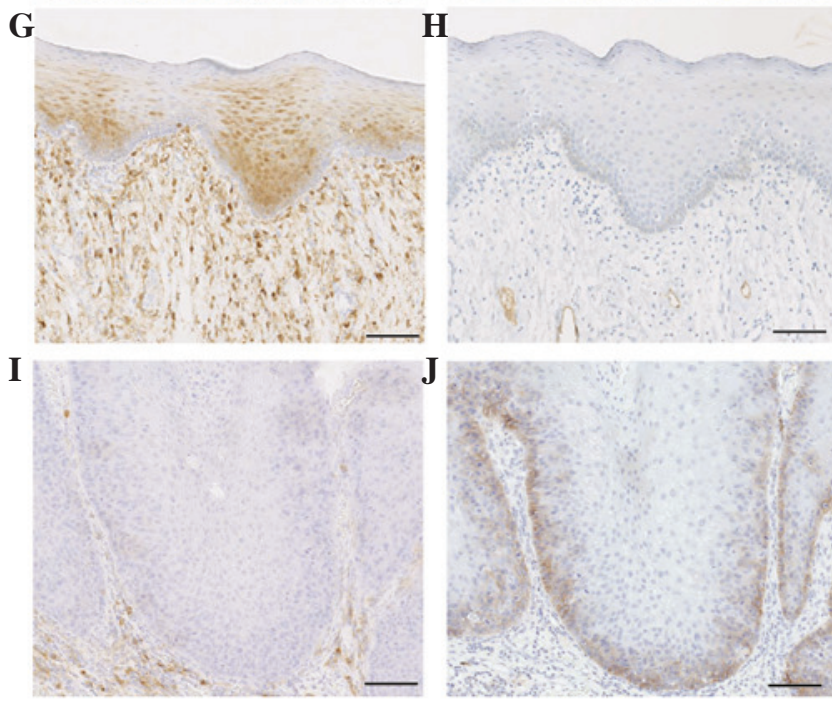

Figure 1. Expression patterns of ALDH1 and podoplanin in serial samples from patients with normal oral epithelium and OL. Negative expression of (A) ALDH1 and (B) podoplanin in normal oral epithelium. Co-expression of (C) ALDH1 and (D) podoplanin in OL. Both (E) ALDH1- and (F) podoplanin-negative OL. (G) ALDH1 positivity, $(\mathrm{H})$ podoplanin negativity, (I) ALDH1 negativity and (J) podoplanin positivity in OL. Scale bar, $100 \mu \mathrm{m}$. ALDH1, aldehyde dehydrogenase 1; OL, oral leukoplakia.

displayed positive expression of ALDH1 and podoplanin, respectively. ALDH1 expression was observed in 21 of the 42 (50\%) patients with UT lesions and in 27 of the $37(73 \%)$ patients with MT lesions ( $\mathrm{P}=0.037)$. Concurrently, podoplanin expression was detected in 22 of the $42(52 \%)$ patients with UT lesions and in 31 of $37(84 \%)$ patients with MT lesions $(\mathrm{P}=0.003)$. Of interest, significant differences in co-expression of ALDH1 and podoplanin $(\mathrm{P}=0.003)$, and expression of either 
Table II. Association between protein expression (ALDH1 and podoplanin) and clinicopathological parameters .

\begin{tabular}{|c|c|c|c|c|c|c|c|c|c|c|c|}
\hline \multirow[b]{3}{*}{ Characteristics } & \multirow[b]{3}{*}{ Patients, $\mathrm{n}$} & \multicolumn{4}{|c|}{ ALDH1 expression } & \multirow[b]{3}{*}{ P-value } & \multicolumn{4}{|c|}{ Podoplanin expression } & \multirow[b]{3}{*}{ P-value } \\
\hline & & \multicolumn{2}{|c|}{ Negative } & \multicolumn{2}{|c|}{ Positive } & & \multicolumn{2}{|c|}{ Negative } & \multicolumn{2}{|c|}{ Positive } & \\
\hline & & $\mathrm{N}$ & $\%$ & $\mathrm{~N}$ & $\%$ & & $\mathrm{~N}$ & $\%$ & $\mathrm{~N}$ & $\%$ & \\
\hline All patients & 79 & 31 & 39 & 48 & 61 & & 26 & 33 & 53 & 67 & \\
\hline Age (years) & & & & & & 0.557 & & & & & 0.475 \\
\hline Mean \pm SD & & $71 \pm 11$ & & $69 \pm 13$ & & & $66 \pm 17$ & & $72 \pm 08$ & & \\
\hline Median & & 72 & & 72 & & & 71 & & 72 & & \\
\hline Range & & $43-90$ & & $23-95$ & & & $23-90$ & & $54-95$ & & \\
\hline Gender & & & & & & 0.925 & & & & & 0.906 \\
\hline Female & 54 & 21 & 68 & 33 & 69 & & 18 & 69 & 36 & 68 & \\
\hline Male & 25 & 10 & 32 & 15 & 31 & & 8 & 31 & 17 & 32 & \\
\hline Site & & & & & & 0.045 & & & & & 0.015 \\
\hline Tongue & 28 & 11 & 35 & 17 & 35 & & 12 & 46 & 16 & 30 & \\
\hline Gingiva & 18 & 2 & 6 & 16 & 33 & & 2 & 8 & 16 & 30 & \\
\hline $\mathrm{BM}$ & 21 & 12 & 39 & 9 & 20 & & 7 & 27 & 14 & 26 & \\
\hline FOM & 5 & 2 & 7 & 3 & 6 & & 0 & 0 & 5 & 9 & \\
\hline Other & 7 & 4 & 13 & 3 & 6 & & 5 & 19 & 2 & 5 & \\
\hline Dysplasia & & & & & & 0.243 & & & & & $<0.001$ \\
\hline LGD & 27 & 13 & 42 & 14 & 29 & & 18 & 69 & 9 & 17 & \\
\hline HGD & 52 & 18 & 58 & 34 & 71 & & 8 & 31 & 44 & 83 & \\
\hline
\end{tabular}

SD, standard deviation; ALDH1, aldehyde dehydrogenase 1; LGD, low-grade dysplasia; HGD, high-grade dysplasia; BM, buccal mucosa; FOM, floor of the mouth.

ALDH1 or podoplanin $(\mathrm{P}=0.013)$, were noted between cases of UT OL and cases of MT OL, respectively (Fig. 2A).

ALDH1 and podoplanin expression, and risk of oral cancer. To estimate the time for malignant transformation of OL, OCFS was analyzed by the Kaplan-Meier method using clinicopathological factors, and ALDH1 and podoplanin expression. In this analysis, the expression levels of ALDH1 and podoplanin, and the grade of dysplasia were observed to be significant indicators using the log-rank test. All findings are summarized in Table III and illustrated in Fig. 3. Patients with positive ALDH1 (Fig. 3A) and podoplanin expression (Fig. 3B) experienced a significantly higher oral cancer incidence than those with negative expression of these proteins. Among the 79 cases, 10 of the 31 (32\%) ALDH1-negative cases and 27 of the $48(56 \%)$ ALDH1-positive cases of OL transformed into oral cancer $(\mathrm{P}=0.006)$, similarly to 6 of the $26(23 \%)$ podoplanin-negative cases and 31 of the $53(58 \%)$ podoplanin-positive cases $(\mathrm{P}=0.010)$. Of interest, co-expression of ALDH1 and podoplanin $(\mathrm{P}<0.001)$ in patients with OL was observed to be strongly prognostic of malignant transformation (Fig. 3C). In addition, in 27 of the 52 (52\%) patients with HGD OL, malignant transformation occurred in a significantly shorter period of time than in those with LGD lesions ( $\mathrm{P}=0.012$ ) (Table III and Fig. 3D).

To evaluate the oral cancer risk in patients with OL, clinicopathological parameters, and ALDH1 and podoplanin expression were analyzed using the Cox proportional hazards model (Table IV). In univariate analysis, the expression levels of ALDH1 $(\mathrm{HR}=2.91 ; 95 \% \mathrm{CI}=1.37-6.92 ; \mathrm{P}=0.005)$ and podoplanin $(\mathrm{HR}=2.95 ; 95 \% \mathrm{CI}=1.32-7.89 ; \mathrm{P}=0.007)$, and the grade of dysplasia $(\mathrm{HR}=2.57 ; 95 \% \mathrm{CI}=1.23-5.77$; $\mathrm{P}=0.011$ ) were significantly associated with an increased risk of malignant transformation. Multivariate analysis was performed to assess the factors that had a significant impact on OCFS in univariate analysis (histology, ALDH1 expression and podoplanin expression). In multivariate analysis, the adjusted HR for malignant transformation was 3.02 for ALDH1 expression (95\% CI=1.39-7.38; P=0.004). Notably, when histology and co-expression of ALDH1 and podoplanin were considered as cofactors, the risk of malignant transformation was considerably higher for OL with positive co-expression of the above proteins compared with OL with negative expression of both proteins ( $\mathrm{HR}=3.64 ; 95 \%$ $\mathrm{CI}=1.71-8.25 ; \mathrm{P}<0.001)$.

To determine the value of ALDH1 and podoplanin for predicting OL malignant transformation, the expression of ALDH1 and podoplanin was evaluated using their PPV. ALDH1 positivity (56\%) and podoplanin positivity (58\%) were associated with higher incidence of oral cancer development compared with the $50.0 \%$ cohort average. Importantly, the expression of both ALDH1 and podoplanin was a strong indicator for oral cancer development (66\% of PPV with marginal significance). In addition, $53 \%$ of the lesions with either ALDH1 or podoplanin expression developed oral cancer (Fig. 2B). 
Table III. Univariate survival analysis by significant prognostic factors using the Kaplan-Meier model.

\begin{tabular}{|c|c|c|c|c|c|c|c|}
\hline \multirow[b]{2}{*}{ Prognostic factors } & \multirow[b]{2}{*}{ Patients, $\mathrm{n}$} & \multicolumn{2}{|c|}{$\begin{array}{l}\text { Patients not } \\
\text { transformed } \\
\text { into } \\
\text { malignancy }\end{array}$} & \multicolumn{2}{|c|}{$\begin{array}{c}\text { Patients } \\
\text { transformed } \\
\text { into } \\
\text { malignancy }\end{array}$} & \multirow{2}{*}{$\begin{array}{l}\text { Median survival } \\
\text { time, months } \\
(95 \% \mathrm{CI})\end{array}$} & \multirow[b]{2}{*}{ P-value } \\
\hline & & $\mathrm{N}$ & $\%$ & $\mathrm{~N}$ & $\%$ & & \\
\hline ALDH1 expression & & & & & & & 0.006 \\
\hline Negative & 31 & 21 & 68 & 10 & 32 & $120(59-125)$ & \\
\hline Positive & 48 & 21 & 44 & 27 & 56 & $51(34-85)$ & \\
\hline Podoplanin expression & & & & & & & 0.010 \\
\hline Negative & 26 & 20 & 77 & 6 & 23 & $102(45-\sim)^{\mathrm{a}}$ & \\
\hline Positive & 53 & 22 & 42 & 31 & 58 & $55(36-85)$ & \\
\hline Dysplasia & & & & & & & 0.012 \\
\hline LGD & 27 & 17 & 63 & 10 & 37 & $102(69-\sim)^{\mathrm{a}}$ & \\
\hline HGD & 52 & 25 & 48 & 27 & 52 & $51(36-69)$ & \\
\hline $\begin{array}{l}\text { Co-expression of proteins } \\
\text { (ALDH1 and podoplanin) }\end{array}$ & & & & & & & $<0.001$ \\
\hline Absent & 44 & 30 & 68 & 14 & 32 & $102(69-125)$ & \\
\hline Present & 35 & 12 & 34 & 23 & 66 & $38(13-55)$ & \\
\hline
\end{tabular}

${ }^{a}$ Upper limit of CI cannot be estimated due to fewer events. CI, confidence interval; ALDH1, aldehyde dehydrogenase 1; LGD, low-grade dysplasia; HGD, high-grade dysplasia.

A

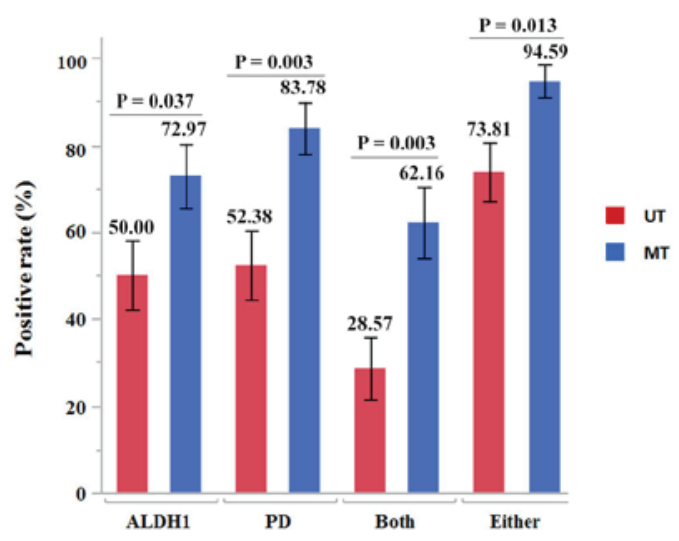

B

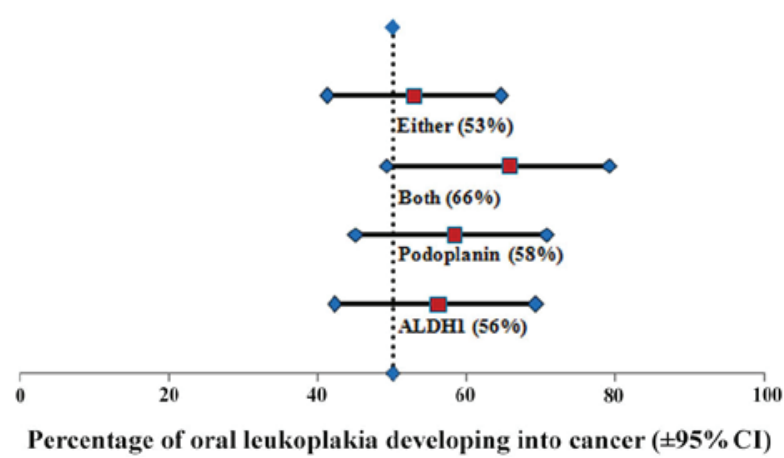

Figure 2. Association between the percentages of positive protein expression and malignant transformation. (A) Percentages of ALDH1- and podoplanin-positive expression in patients with UT lesions and patients with MT lesions of OL. (B) Point-prevalence analysis of the incidence of oral cancer development from OL with ALDH1 and podoplanin expression as PPVs and the 95\% CI. The PPVs of ALDH1 expression, podoplanin expression and protein co-expression were 56,58 and $66 \%$, respectively. The vertical dashed line indicates the 50\% of this cohort that developed malignancy. ALDH1, aldehyde dehydrogenase 1; PD, podoplanin; MT, malignant transformed; UT, untransformed; PPV, point-prevalence value; CI, confidence interval; OL, oral leukoplakia. 
Table IV. Cox proportional hazards regression models to estimate cancer development.

\begin{tabular}{lrr}
\hline Characteristics & HR & $95 \%$ CI \\
\hline Univariate analysis & 1.44 & $0.74-2.89$ \\
Age (>70 vs. $\leq 70$ years) & 1.58 & $0.72-3.29$ \\
Gender (male vs. female) & 2.57 & $1.23-5.77$ \\
Histology (HGD vs. LGD) & 2.91 & $1.37-6.92$ \\
ALDH1 expression (positive vs. negative) & 2.95 & $1.32-7.89$ \\
Podoplanin expression (positive vs. negative) & 1.55 & 0.239 \\
Multivariate analysis (histology, ALDH1 and podoplanin expression) & 0.011 \\
Histology (HGD vs. LGD) & 3.02 & 0.005 \\
ALDH1 expression (positive vs. negative) & 2.62 & $1.39-7.38$ \\
Podoplanin expression (positive vs. negative) & $1.04-7.59$ \\
Multivariate analysis (histology, and co-expression of ALDH1 and podoplanin) & 0.81 \\
Histology (HGD vs. LGD) & 1.61 & 0.004 \\
Co expression of ALDH1 and podoplanin & 3.64 \\
Present vs. absent & 0.039 \\
\hline
\end{tabular}

HR, hazard ratio; CI, confidence interval; ALDH1, aldehyde dehydrogenase 1; LGD, low-grade dysplasia; HGD, high-grade dysplasia.

A

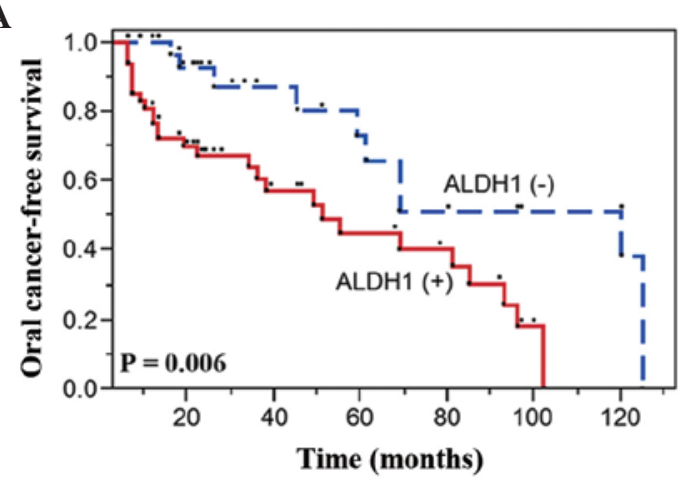

C

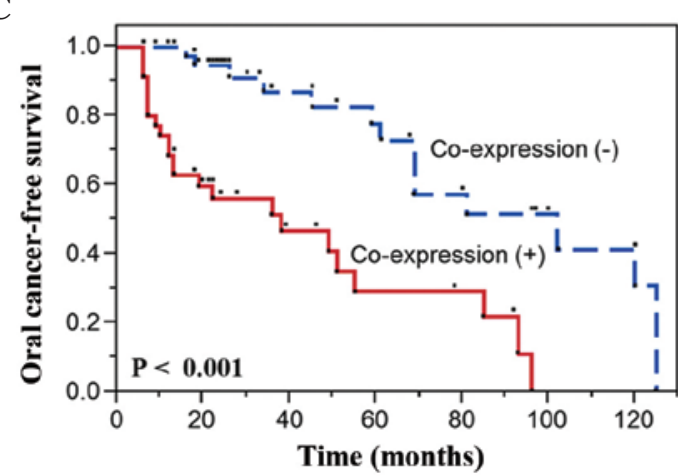

B

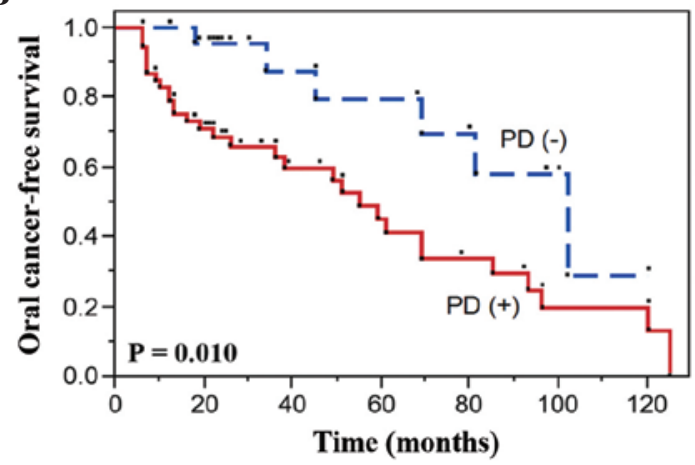

D

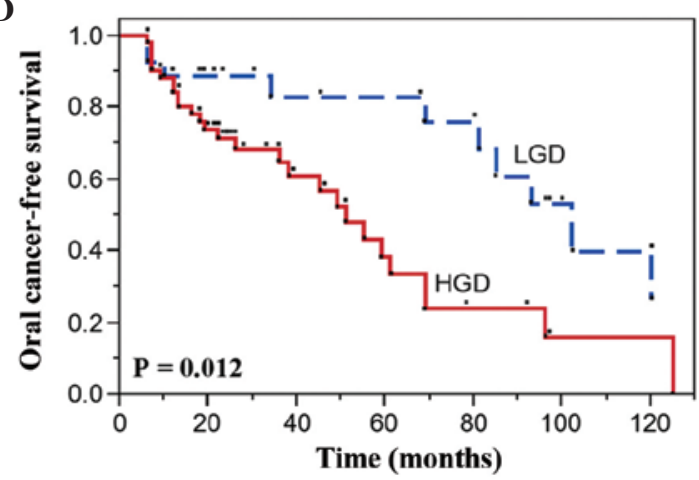

Figure 3. Kaplan-Meier oral cancer-free survival curves according to (A) ALDH1 negativity and positivity; (B) podoplanin negativity and positivity; (C) presence or absence of co-expression of ALDH1 and podoplanin; and (D) degree of dysplasia. Comparisons were performed using the log-rank test. ALDH1, aldehyde dehydrogenase 1; PD, podoplanin; LGD, low-grade dysplasia; HGD, high-grade dysplasia.

\section{Discussion}

The current study attempted to determine the usefulness of ALDH1 and podoplanin expression for predicting the transformation of OL with varying grades of dysplasia into OSCC, and to assess the clinical implications of ALDH1 and podoplanin expression in patients with OL on the basis of the cancer stem cell theory. Braakhuis et al (29) proposed a patch-field carcinoma progression model of oral cancer, and hypothesized that oral cancer development started with a 'patch stem cell' that developed into an expanding subpopulation of stem cells escaping growth control, eventually resulting in malignant transformation.

ALDH1 has been demonstrated to be a cancer stem cell marker in several solid tumor types, including OSCC $(11,14)$. 
ALDH1 was reported to serve a crucial role in maintaining the self-renewal properties and tumorigenicity of HNSCC-derived cancer stem cells (30), and its immunoexpression is associated with a poor prognosis of patients with $\operatorname{HNSCC}(19,20)$. Visus et al (18) observed ALDH1 overexpression in samples of oral dysplasia and HNSCC, and suggested that it was a marker for distinguishing malignant from premalignant cells in HNSCC, in addition to being an essential epitope for developing ALDH1-based vaccines for HNSCC therapy. The present study observed that the overexpression of ALDH1 in OL samples was a significant predictor of malignant transformation.

Although the role of podoplanin in carcinogenesis is still a matter of debate, it has been reported that podoplanin is expressed in approximately $90 \%$ of OSCCs (21). Podoplanin is involved in the remodeling of the cell cytoskeleton mediated by actin, and may promote cell invasion by increasing cell motility (22). Podoplanin-positive cells in the epithelial layers may represent upward clonal expansion of stem cells during carcinogenesis and oral disorders, and such clonal expansion may represent a higher risk of malignant transformation. Kawaguchi et al (26) reported that podoplanin was a marker of malignant transformation in OL and other oral precancerous lesions. In agreement with that study, the present study also demonstrated that podoplanin expression in OL was associated with an increased risk of malignant transformation. Other studies also support a relevant role for podoplanin in early oral tumorigenesis, even though podoplanin expression alone may not be sufficient to promote carcinogenesis $(25,26,31)$.

In the present study, immunohistochemical staining of both ALDH1 and podoplanin was performed to evaluate the oral cancer risk in patients with OL. Kaplan-Meier analysis indicated a significant impact for the 5-year OCFS rate, and demonstrated that $55 \%$ of patients with ALDH1 positivity developed OSCC compared with $27 \%$ of those with ALDH1 negativity. In addition, $55 \%$ of patients with podoplanin positivity developed OSCC compared with $20 \%$ of those with podoplanin negativity. Multivariate analysis revealed that the expression of ALDH1 and podoplanin was associated with a 3.02- and 2.62-fold increased risk of transformation, respectively. Taken together, these data not only support the potential importance of ALDH1 and podoplanin in oral carcinogenesis, but also suggest that both proteins may be used as biomarkers for evaluating the malignant transformation risk in oral premalignancy. However, contrary to previous findings, $10(32 \%)$ ALDH1- and 6 (23\%) podoplanin-negative patients also developed cancers (although later than expression-positive patients) in the present study. Their delayed but cancerous transformation can be attributed to the following possible reasons: The lesions were biopsied prior to when the abnormality occurred, or those cancers originated from lesions not clinically visible at the time of biopsy, which therefore remained unexamined. Another possibility is that the biopsies were obtained from different clonal sites than those from which the cancers eventually developed (26).

Although there is general agreement that the rate of malignant transformation increases with the severity of the dysplasia, certain authors did not observe a significant association between epithelial dysplasia and malignant transformation (32). The present study demonstrated that the grade of dysplasia was a significant risk factor for malignant transformation; however, the prognostic values of ALDH1 and podoplanin proteins were superior to that of histological grading, which was associated with a 1.55 -fold increased risk. Furthermore, significant differences in the co-expression of ALDH1 and podoplanin proteins, and histological examination were also noted in malignant transformation of OL. This suggested that the co-expression of both biomarkers may be more informative than the histological examination alone. Therefore, immunohistochemical staining of ALDH1 and podoplanin could help to augment the predictability and reliability of cancer risk assessment for OL in association with histopathologic assessment of epithelial dysplasia.

Cancer of the oral cavity results in severe morbidity, limited quality of life and short overall survival, and there is a strong requirement to understand oral carcinogenesis and to establish accurate and reliable predictors of oral cancer risk $(1-3,33)$. The interpretation of the protein expression of significant biomarkers is relatively simple in routine/diagnostic laboratories. Therefore, immunohistochemical staining of biomarkers is promising for the evaluation of oral cancer risk (17).

To the best of our knowledge, the present report is the first study investigating the combined expression of ALDH1 and podoplanin for oral cancer risk assessment in patients with OL. Our data revealed that positive expression of ALDH1 and podoplanin in OL was significantly associated with the risk of malignant transformation and, consequently, such leukoplakia should be followed carefully. However, further studies are required to fully define the functional roles of these biomarkers in oral cancer initiation and disease progression.

In summary, ALDH1 and podoplanin can be used as biomarkers for risk assessment of oral malignant transformation in patients with OL.

\section{Acknowledgements}

The authors thank Mr. Yohei Murayama and Ms. Tomomi Takahashi (Support Section for Education and Research, Hokkaido University Graduate School of Dental Medicine, Sapporo, Japan) for their technical assistance.

\section{References}

1. Zini A, Czerninski R and Sgan-Cohen HD: Oral cancer over four decades: Epidemiology, trends, histology, and survival by anatomical sites. J Oral Pathol Med 39: 299-305, 2010.

2. Ganly I, Patel S and Shah J: Early stage squamous cell cancer of the oral tongue-clinicopathologic features affecting outcome. Cancer 118: 101-111, 2012.

3. Warnakulasuriya S: Living with oral cancer: Epidemiology with particular reference to prevalence and life-style changes that influence survival. Oral Oncol 46: 407-410, 2010.

4. Papadimitrakopoulou VA, Hong WK, Lee JS, Martin JW, Lee JJ, Batsakis JG and Lippan SM: Low-dose isotretinoin versus beta-carotene to prevent oral carcinogenesis: Long-term follow-up. J Natl Cancer Inst 89: 257-258, 1997.

5. Silverman S Jr, Gorsky M and Lozada F: Oral leukoplakia and malignant transformation. A follow-up study of 257 patients. Cancer 53: 563-568, 1984.

6. Lee JJ, Hong WK, Hittelman WN, Mao L, Lotan R, Shin DM, Benner SE, Xu XC, Lee JS, Papadimitrakopoulou VM, et al: Predicting cancer development in oral leukoplakia: Ten years of translational research. Clin Cancer Res 6: 1702-1710, 2000.

7. Sayed SI, Dwivedi RC, Katna R, Garg A, Pathak KA, Nutting CM, Rhys-Evans P, Harrington KJ and Kazi R: Implications of understanding cancer stem cell (CSC) biology in head and neck squamous cell cancer. Oral Oncol 47: 237-243, 2011 
8. Zhang Z, Filho MS and Nör JE: The biology of head and neck cancer stem cells. Oral Oncol 48: 1-9, 2012.

9. Krishnamurthy S and Nör JE: Head and neck cancer stem cells. J Dent Res 91: 334-340, 2012.

10. Gires O: Lessons from common markers of tumor-initiating cells in solid cancers. Cell Mol Life Sci 68: 4009-4022, 2011.

11. Chen YC, Chen YW, Hsu HS, Tseng LM, Huang PI, Lu KH, Chen DT, Tai LK, Yung MC, Chang SC, et al: Aldehyde dehydrogenase 1 is a putative marker for cancer stem cells in head and neck squamous cancer. Biochem Biophys Res Commun 385 307-313, 2009.

12. Wicki A and Christofori G: The potential role of podoplanin in tumor invasion. Br J Cancer 96: 1-5, 2007.

13. Marcato P, Dean CA, Giacomantonio CA and Lee PW: Aldehyde dehydrogenase: Its role as a cancer stem cell marker comes down to the specific isoform. Cell Cycle 10: 1378-1384, 2011.

14. Clay MR, Tabor M, Owen JH, Carev TE, Bradford CR, Golf GT, Wicha MS and Prince ME: Single-marker identification of head and neck squamous cell carcinoma cancer stem cells with aldehyde dehydrogenase. Head Neck 32: 1195-1201, 2010.

15. Chen YC, Chang CJ, Hsu HS, Chen YW, Tai LK, Tseng LM, Chiou GY, Chang SC, Kao SY, Chiou SH and Lo WL: Inhibition of tumorigenicity and enhancement of radiochemosensitivity in head and neck squamous cell cancer-derived ALDH1-positive cells by knockdown of Bmi-1. Oral Oncol 46: 158-165, 2010.

16. Chen C, Wei Y, Hummel M, Hoffmann TK, Gross M, Kaufmann AM and Albers AE: Evidence for epithelial-mesenchymal transition in cancer stem cells of head and neck squamous cell carcinoma. PLoS One 6: e16466, 2011.

17. Liu W, Wu L, Shen XM, Shi LJ, Zhang CP, Xu LQ and Zhou ZT: Expression patterns of cancer stem cell markers ALDH1 and CD133 correlate with a high risk of malignant transformation of oral leukoplakia. Int J Cancer 132: 868-874, 2013.

18. Visus C, Ito D, Amoscato A, Macieiewska-Franczak M, Abdelsalem A, Dhir R, Shin DM, Donnenbera VS, Whiteside TL and DeLeo AB: Identification of human aldehyde dehydrogenase 1 family member A1 as a novel CD8+ T-cell-defined tumor antigen in squamous cell carcinoma of the head and neck. Cancer Res 67: 10538-10545, 2007.

19. Chen YW, Chen KH, Huang PI, Chen YC, Chiou GY, Lo WL, Tseng LM, Hsu HS, Chang KW and Chiou SH: Cucurbitacin I suppressed stem-like property and enhanced radiation-induced apoptosis in head and neck squamous carcinoma-derived CD44(+) ALDH1(+) cells. Mol Cancer Ther 9: 2879-2892, 2010.

20. Luo WR, Gao F, Li SY and Yao KT: Tumor budding and the expression of cancer stem cell marker aldehyde dehydrogenase 1 in nasopharyngeal carcinoma. Histopathology 61: 1072-1081, 2012.

21. Deng S, Yang X, Lassus H, Liang S, Kaur S, Ye Q, Li C, Wang LP, Roby KF, Orsulic S, et al: Distinct expression levels and patterns of stem cell marker, aldehyde dehydrogenase isoform 1 (ALDH1), in human epithelial cancers. PLoS One 5: e10277, 2010.
22. Kahn HJ and Marks A: A new monoclonal antibody, D2-40, for detection of lymphatic invasion in primary tumors. Lab Invest 82: 1255-1257, 2002.

23. Yuan P, Temam S, El-Naggar A, Zhou X, Liu DD, Lee JJ and Mao L: Over expression of podoplanin in oral cancer and its association with poor clinical outcome. Cancer 107: 563-569, 2006.

24. Shi P, Liu W, Zhou ZT, He QB and Jiang WW: Podoplanin and ABCG2: Malignant transformation risk markers for oral lichen planus. Cancer Epidemiol Biomarkers Prev 19: 844-849, 2010.

25. Saintigny P, El-Naggar AK, Papadimitrakopoulou V, Ren H, Fan YH, Feng L, Lee JJ, Kim ES, Hong WK, Lippman SM and Mao L: DeltaNp63 over expression, alone and in combination with other biomarkers, predicts the development of oral cancer in patients with leukoplakia. Clin Cancer Res 15: 6284-6291, 2009.

26. Kawaguchi H, El-Naggar AK, Papadimitrakopoulou V, Ren H, Fan YH, Feng L, Lee JJ, Kim E, Hong WK, Lippman SM and Mao L: Podoplanin: A novel marker for oral cancer risk in patients with oral premalignancy. J Clin Oncol 26: 354-360, 2008.

27. Pindborg JJ, Reichart PA, Smith CJ and Van der Waal I (eds): Histological Typing of Cancer and Precancer of the Oral Mucosa. World Health Organization. Springer, Berlin, pp25-26, 1997.

28. Habiba U, Kitamura T, Yanagawa-Matsuda A, Hida K, Higashno F, Ohiro Y and Shindoh M: Cytoplasmic expression of HuR may be a valuable diagnostic tool for determining the potential for malignant transformation of oral verrucous borderline lesions. Oncol Rep 31: 1547-1554, 2014.

29. Braakhuis BJ, Leemans CR and Brakenhoff RH: A genetic progression model of oral cancer: Current evidence and clinical implications. J Oral Patho Med 33: 317-322, 2004.

30. Wu A, Luo W, Zhang Q, Yang Z, Zhang G, Li S and Yao K: Aldehyde dehydrogenase 1, a functional marker for identifying cancer stem cells in human nasopharyngeal carcinoma. Cancer Lett 330: 181-189, 2013.

31. Inoue H, Miyazaki Y, Kikuchi K, Yoshida N, Ide F, Ohmori Y, Tomomura A, Sakashita H and Kusama K: Podoplanin expression during dysplasia-carcinoma sequence in the oral cavity. Tumor Biol 33: 181-194, 2012.

32. Holmstrup P, Vedtofte P, Reibel J and Stoltze K: Long term treatment outcome of oral premalignant lesions. Oral Oncol 42: 461-474, 2006.

33. Carlos de Vicente J, Rodrigo Palbo J, Santamarta-Rodriguez T, Lequerica-Fernández P, Allonca E and García-Pedrero JM: Podoplanin expression in oral leukoplakia: Tumorigenic role. Oral Oncol 49: 598-603. 2013. 